\title{
A TEST OF SEVERAL HYPOTHESES FOR THE DETERMINATION OF SEED NUMBER IN AMELANCHIER ARBOREA, USING SIMULATED PROBABILITY DISTRIBUTIONS TO EVALUATE DATA ${ }^{1}$
}

\author{
David L. Gorchov and George F. Estabrook \\ Department of Biology, University of Michigan, Ann Arbor, Michigan 48109-1048
}

\begin{abstract}
A B S T R A C T
The number of seeds per fruit is variable within Amelanchier arborea trees. Because $A$. arborea flowers are five-carpellate and each carpel contains two ovules, we were able to use the pattern of seed maturation within fruits to test whether the failure of some ovules to develop into seeds is determined by mechanisms operating at the level of carpels, such as stigma-clogging, or by mechanisms operating at the level of ovules, such as ovule infertility. The presence of one-, two-, and zero-seeded carpels demonstrated that the number of ovules developing into seeds was not due entirely to carpel-level phenomena. In order to test the hypothesis of carpel independent seed development, without the assumption that all ovules have the same probability of developing into seeds, it was necessary to use simulation, since no conventional statistical models were appropriate. Analysis of this simulation allowed us to reject carpel independent processes as the only determinant of seed number. A mixed model of seed development, in which some carpels fail entirely and ovules in the remaining carpels develop equiprobably, was shown to be consistent with the data.
\end{abstract}

MANY, IF NOT MOST, plants have a variable number of seeds per fruit. For these plants, seed number is one component of yield or fitness that the plant can adjust in response to environmental conditions (Harper, 1977). In some species seed number has a strong effect on fruit size (Wareing and Phillips, 1978, 123), pulp : seed ratio (Herrera, 1981), mass : volume ratio (Augspurger and Hogan, 1983), or fruit developmental time (Lee and Bazzaz, 1982), all of which can have consequences for seed dispersal.

Pollination, resources, and seed predation have all been proposed as mechanisms for the determination of seed number per fruit, as well as other components of plant reproductive output (for reviews, see Bierzychudek, 1981; Stephenson, 1981; Rathcke, 1983; Bawa and Webb, 1984). Most studies to date have used pollen or resource augmentation to test whether these factors limit seed number. A recent review (Snow, 1986) showed that seed number is related to pollination intensity in only a minority of species investigated to date, and these are mostly species with a very large number of ovules per flower. Here we show that analysis of the distribution of seeds among the carpels

\footnotetext{
' Received for publication 10 December 1986; revision accepted 26 March 1987.

We thank two anonymous reviewers for helpful comments. Research support to DLG from the University of Michigan Museum of Zoology and Rackham School of Graduate Studies is gratefully acknowledged.
}

of a fruit can be an additional tool for distinguishing among competing hypotheses.

MATERIALS AND METHODS-Amelanchier arborea (Michx. f.) Fern. (Downy Serviceberry) is a small tree of eastern North America that bears fleshy fruits containing one to ten filled seeds. Unlike some congeners, it is self-incompatible and not apomictic (Robinson, 1982; Gorchov, 1985). As in other members of the Rosaceous subfamily Maloideae, the gynoecium consists of five carpels each made up of a single stigma, a style, and two ovules (see Campbell et al., 1985, and Olson and Steeves, 1982, for detailed descriptions of other Amelanchier species). Thus, each flower should have the potential to produce fruits with ten seeds. Fruits usually do contain ten "seeds," but the number of these that are filled with an embryo ranges from one to ten and is always variable among fruits within a plant. Some of the remaining seeds are of nearly full length but contain no embryo ("empty seeds"), but most are very small. The latter ("undeveloped seeds") are not necessarily derived from fertilized ovules: they can develop from ovules in carpels that had their styles pinched off before pollination (Gorchov, 1987).

Filled seed number in $A$. arborea is negatively correlated with the number of days from fruit initiation to ripening, resulting in a within-plant ripening phenology that is much more asynchronous than the flowering phenology 
TABLE 1. Hypotheses for the determination of flled seeds per A. arborea fruit. Rej. = rejected based on manipulation experiments (Gorchov, 1987). Seed predation (3) reduces filled seed number in some fruit, but flled seed number is variable among undamaged fruits

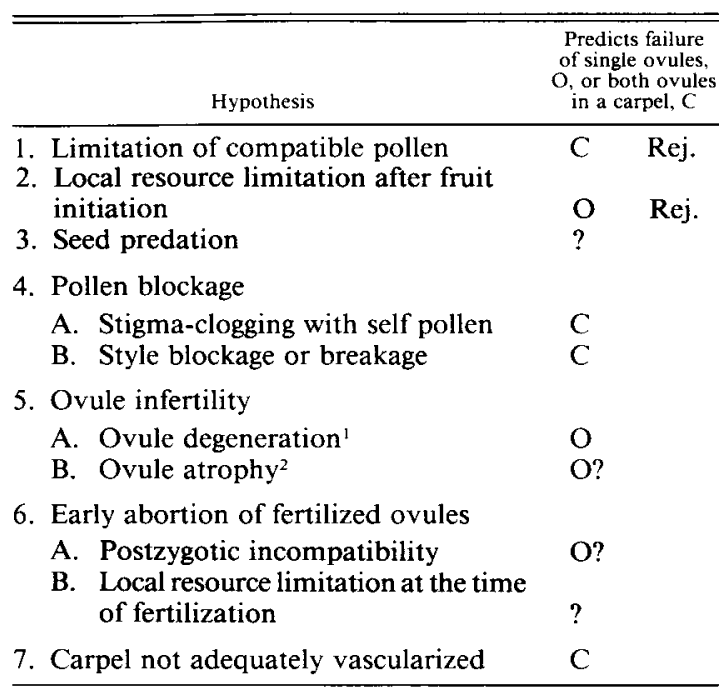

' Premature cessation of development.

${ }^{2}$ Senescence of unfertilized ovules.

(Gorchov, 1985). Amelanchier arborea fruits are eaten by a variety of birds and mammals, many of which are seed dispersal agents (Robinson, 1986; Gorchov, 1987), and this asynchronous ripening may be an adaptation to avoid satiation of seed dispersers (Thompson and Willson, 1979).

Seed predators (primarily moth larvae) reduce the number of filled seeds in some fruits, but filled seed number varies even among undamaged fruit (plant means range from 3.8 to 8.3 and with SD from 1.4 to 2.2 ). To test whether seed number in undamaged fruits is determined by pollen or by resources, field manipulations were carried out (Gorchov, 1987). Supplemental xenogamous pollination did not affect the mean or variance of seed number, indicating seed number is not limited by the amount of compatible pollen arriving at the stigmas. Similarly, manipulations of local resource levels at fruit initiation by defoliation, girdling, and fruit thinning did not affect seed number, although they did affect fruit set and seed weight.

These results suggest that seed number in $A$. arborea is determined by neither pollen nor resources. Several post hoc hypotheses for seed number determination that are consistent with these data have been proposed (Gorchov, 1987 ). Because $82 \%$ of styles with any pollen tubes have a large number of them (Gorchov, 1987) and each style delivers pollen to only one carpel, style/pollination related hypotheses predict that both ovules in a carpel will share the same fate: either develop or fail to develop. Hypotheses that attribute ovule failure to genetic or developmental factors predict that failure of an ovule is independent of failure of the other ovule in the carpel (Table 1). While the individual hypotheses might be testable by future experimental work, the two classes of hypotheses are potentially distinguishable by patterns of seed development within carpels.

Carpel walls can be discerned in mature fruits if they are dissected carefully. It is easier to distinguish the carpel walls in immature fruits. This was done by one of us (DLG) for a set of fruits collected from four individual plants growing in an oak-hickory woods at the E. S. George Reserve, southeastern Michigan.

Four inflorescences on each of the plants were thinned to two flowers at peak bloom (2224 April 1985). On 4 May four other inflorescences on each individual were thinned to two developing fruits, and the leaves on each of these shoots were removed. Fruits from both the defoliation and control treatments were collected one to two weeks before ripening began in the population (18 May for two plants, 24 May for the other two). By this time seeds were approximately full-sized but had not yet acquired hardened seed coats, and fruit abortion was more than $90 \%$ complete.

For each fruit the number of carpels containing two, one, and zero developed seeds were counted. (Developed seeds include both filled and empty seeds, since it is possible that some seeds that appear filled at this point in development abort before fruit ripening.) A few fruits (11\%) had four rather than five carpels and were not included in this analysis. The frequency of these four-carpellate fruits did not differ significantly between the defoliation and control treatments. There was also no significant difference between the treatments in the number of developed seeds per undamaged, five-carpellate fruit. This lack of difference between the treatments justified pooling the seed distribution data.

HYPOTHESES - If seed development or failure was entirely determined by a carpel-level mechanism (Table 1), then we would expect each carpel to have either two or zero seeds. However, a large number of carpels (32\%) contained one developed and one undeveloped seed (Table 2), indicating that failure of ovules to develop is not solely dependent on carpellevel processes.

If, instead, one of the ovule-level mechanisms (Table 1) is valid, we would expect seed 
development to be independent of carpel membership. It is difficult to predict the distribution of seeds in carpels that would be expected under such a hypothesis, unless simplifying assumptions are made. One such simplifying assumption is that all ovules have the same probability, $P$, of developing into a seed. At least two distinct biological processes could lead to this assumption being valid. First, ovule failure may be due to some developmental problem (e.g., hypotheses $5 \mathrm{~A}, 5 \mathrm{~B}$, and $6 \mathrm{~A}$, Table 1) that is equally likely to affect any ovule. Second, resource allocation to different flowers or fruits may be adjusted by the plant so as to maintain a constant $P$ despite differences in local resource levels. This would result in a binomial distribution of seed number per fruit. The latter pattern could be adaptive if, for example, it resulted in a variance in seed number that, through the dependence of fruit developmental time on seed number (Gorchov, 1985), generated an optimal degree of ripening synchrony.

Under the simplifying assumption of a globally constant $P$ the expected frequency of two-, one-, and zero-seeded carpels is given by the binomial distribution. The observed distribution has significantly fewer one-seeded fruits than expected (Table 2), allowing us to reject the hypothesis that ovules develop into seeds with a constant probability.

Two classes of explanations remain for the development of ovules into seeds. First, development of ovules into seeds may occur independently (with respect to carpels) within fruits, but the probability that an ovule develops into a seed may not be the global constant stated in the above hypothesis, but rather may vary among fruits or plants. This model would be consistent with any of the ovule-level hypotheses in Table 1. For example, flower primordia experiencing different resource levels may complete development of ovules with differing probabilities, but two ovules in the same carpel would be as likely to both fail to differentiate as any two ovules in the same fruit. In this case, the observed number of carpels with two, one, or zero seeds would reflect mixed samples from two or more binomial distributions with different seed development probabilities corresponding to differing intensities of the hypothesized ovule-level explanations in Table 1. One property of mixed binomial distributions is that the observed number of one-seeded carpels is lower than predicted by the single binomial distribution whose global probability is estimated from the data. Thus the trend in our data away from the number of single-seeded carpels predicted by the global
TABLE 2. Number of carpels with two, one, and zero developed seeds observed in a sample of immature fruits and expected based on a constant ovule development probability of $(91 \times 2+69) / 215=0.58$ that is random with respect to position. The observed distribution differs significantly from the expected (Goodness-of-fit $\chi^{2}=24.7, d f=2, P<0.001$ )

\begin{tabular}{ccr}
\hline & \multicolumn{2}{c}{$\begin{array}{c}\text { Carpels containing this number } \\
\text { of developed seeds }\end{array}$} \\
\cline { 2 - 3 } Developed seeds & Observed & Expected \\
\hline 2 & 91 & 73.3 \\
1 & 69 & 104.5 \\
0 & $\underline{55}$ & $\underline{37.3}$ \\
Total & 215 & 215.1 \\
\hline
\end{tabular}

binomial process rejected in Table 2 is consistent with this first class of remaining explanations.

A second possibility is that ovule failure is partly, but not completely, dependent on carpel membership. This could involve two distinct but simultaneous processes, one operating at the level of carpels to produce failure of both ovules and the second at the level of ovules independent of carpels. Partial dependence on carpels could alternatively be due to a process, such as fungal infection, that tended to affect neighboring ovules, whether they were in the same carpel or not. Either process would increase the predicted number of empty carpels and thus predict that the number of one-seeded carpels will be lower than expected by the constant $P$ hypothesis rejected in Table 2 . Because both classes of remaining explanations are consistent with (and thus cannot be distinguished by) the way in which the hypothesis of globally constant probability of development is rejected, we must seek a more powerful means to distinguish these two classes.

Although the former ovule-based explanation does not retain the assumption of a globally constant probability of seed development, it still assumes that seed development of one ovule is independent of seed development of another ovule. An important consequence of this assumption is that once given that a particular fruit has a particular number, $N$, of seeds, those seeds will have developed from any $N$ of the 10 ovules with equal probability, independent of carpel membership. By contrast, the latter, carpel-dependent, explanation explicitly requires a violation of this independence assumption. Thus, we can take the independence assumption of the ovule-level hypotheses and derive distributions for statistics to test it. Its rejection will specifically implicate carpel-level explanations.

A difficulty arises in testing this hypothesis: 


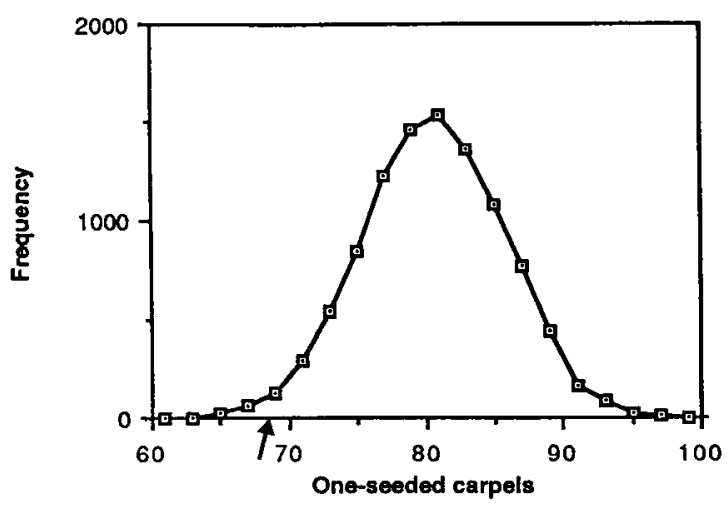

Fig. 1. Frequency distribution of number of carpels with one seed resulting from 10,000 replications of a program simulating the assignment of seeds into carpels based on seed numbers in an observed set of 43 Amelanchier arborea fruit. Arrow indicates the number of one-seeded carpels in the observed set of fruit (69). Realized significance of this number of one-seeded carpels is 0.023 .

what distribution of seeds in carpels does it predict? To answer this question, we generated these distributions by computer simulation. We specified the number of fruits with each different seed number. Then, for each fruit in the data set to be simulated, we assigned the seeds to the ovules equiprobably, without regard to carpels. Afterward, in the simulated data set, the number of carpels with two, one, and zero seeds was observed and recorded. This process was replicated 10,000 times to obtain frequency distributions for the number of carpels with two, one, and zero seeds predicted by the ovule based hypotheses.

RESULTS AND DISCUSSION-Figure 1 shows the frequency distribution of one-seeded carpels among 43 fruits for which seed number per fruit was specified by the data and seeds were assigned to carpels equiprobably, based on 10,000 replications of the simulation. The observed number of one-seeded carpels, 69, lies at the lower tail of this distribution; only $2.3 \%$ of the replicates resulted in 69 or fewer one-seeded carpels. Similarly, the observed number of zero-seeded carpels (55) was matched or exceeded in only $2.3 \%$ of the runs, and the observed number of two-seeded carpels (91) was matched or exceeded in $2.3 \%$. Thus, the observed data include fewer one- and more zero- and two-seeded carpels than expected under the assumption of ovule failure independent of carpel membership, even after relaxing the assumption of globally constant ovule failure probability.

These results demonstrate that the assumption that the failure of an ovule is independent of the fate of the other ovule in the carpel does not hold for $A$. arborea. Seed number per fruit cannot be determined solely by ovule-level phenomena. This finding, when combined with the initial observation that a third of the carpels contain one seed, implies that ovule failure is partially, but not completely, dependent on carpel membership. Failure of an ovule to develop into a seed therefore is either determined by a mechanism that tends to but does not always affect both ovules in a carpel (e.g., fungal infection) or it is partly due to a mechanism operating at the level of carpels and partly due to a mechanism operating at the level of ovules.

By simulating probability distributions for numbers of carpels with two, one, or zero seeds under the specific conditions of independence of ovule development, and of the numbers of fruits with the numbers of seeds occurring in the data, we have shown that the tendency of two ovules in a carpel to develop or fail together is statistically significant. Because experimentally added xenogamous pollen failed to increase seed set in naturally pollinated flowers (but produced seed set in bagged flowers) and $89 \%$ of styles from naturally pollinated flowers contained pollen tubes (Gorchov, 1987), it is unlikely that zero-seeded carpels are the result of inadequate pollen delivery. Therefore, it is likely that much of the style-mediated effect is through the failure of styles by improper development, clogging by incompatible pollen, breakage, or some other cause of style failure. If carpels in which both seeds had failed due to style or whole carpel effects could be removed from the data, the remaining carpels could be used to test ovule-related hypotheses. In particular, with ovules developing independently of carpels, the hypothesis of a constant global probability of development could be tested. Of course, we cannot tell which empty carpels failed for style/carpel reasons and which just happened to contain ovules that independently did not develop. However, we can hypothesize a constant global probability of development for ovules in carpels that did not fail for carpel/style reasons, and see how well our data can be made to fit.

For each proportion, $f$, of zero-seeded carpels attributable to carpel failure, we calculated how well the remaining distribution of carpels fit a binomial distribution. We found that if $f=76 \%$ of the zero-seeded carpels were attributable to carpel-level failure, then the remaining carpels (91 two-seeded, 69 one-seeded, and 13 seedless) best fit a binomial distribution $\left(\chi^{2}=0.0003\right)$. This corresponds to an overall carpel failure rate of $20 \%$. Thus it is possible that about a fifth of the carpels fail for carpel/style reasons and in the remainder seeds develop with a constant global probability. 
Impossible hypotheses can be rejected without statistical argument. The power of statistical argument is to determine how likely the data are given that a possible hypothesis is true; data very unlikely under this condition argue against the hypothesis. To assess this likelihood in our case, we need a distribution for chi-square under our unusual post hoc procedure of successively removing empty carpels until goodness-of-fit chi-square is minimized. The classical distribution of chi-square for $\mathrm{df}=2$ is clearly not appropriate. To determine the distribution of chi-square appropriate for our application, we simulated our post hoc hypothesis-fitting activity with a computer.

Our computer program determined every possible combination of two-seeded, one-seeded, and seedless carpels that added to a total equal to a given number of carpels (in this example, 215, the number of carpels in the data set). For each of these combinations $(22,791$ in this case), the program deleted seedless carpels (i.e., attributed them to carpel failure), one at a time, until all seedless carpels were deleted. At each step in this progressive deletion, the chi-square goodness-of-fit to a binomial distribution was calculated; then the program found the minimum value for each of these combinations. These 22,791 minimum chisquare values provided a distribution with which to compare our observed chi-square of 0.0003 . The proportion of these minima falling below our observed value, and hence the realized significance of our result, was $P=0.13$.

In order to explore whether this result was affected by the inclusion of combinations in which the total number of seeds was very different than the observed, we repeated this procedure confining the analysis to those combinations in which the total number of seeds was close to the observed. In our data $58 \%$ of the ovules developed into seeds, so we confined this analysis to those combinations of two-, one-, and zero-seeded carpels that contained $50-70 \%$ seeds. There were 7,417 combinations that met this criteria, and $8 \%$ had minimum chi-square values below our observed value of 0.0003 .

This analysis shows that the good fit of our data to the binomial distribution, after a flexible proportion of seedless carpels was attributed to carpel failure, was moderately surprising. This enhances the plausability that seed number in $A$. arborea is determined by two mechanisms: some carpels fail entirely and those ovules in the remaining carpels are equally likely to develop into seeds.

We hope that this report will provide other investigators with a statistical framework for distinguishing between hypotheses for the pattern of seed development within fruits or other similar processes. A critical advantage of this sort of simulation is that it allows for the testing of specific hypotheses without requiring assumptions that all other things are equal.

Readers wishing to receive Pascal source code for these two programs on $5 \frac{1 / 4}{4}$ floppy disk in IBM/PC compatible format are invited to mail us such a formatted disk.

\section{LITERATURE CITED}

Augspurger, C. K., AND K. P. Hogan. 1983. Wind dispersal of fruits with variable seed number in a tropical tree (Lonchocarpus pentaphyllus): Leguminosae. Amer. J. Bot. 70: 1031-1037.

BAwA, K. S., AND C. J. WEBB. 1984. Flower, fruit, and seed abortion in tropical forest trees: implications for the evolution of paternal and maternal reproductive patterns. Amer. J. Bot. 71: 736-751.

BIERZYCHUDEK, P. 1981. Pollinator limitation of plant reproductive effort. Amer. Naturalist 117: 838-840.

Campbell, C. S., C. W. Greene, B. F. Neubauer, and J. M. Higgins. 1985. Apomixis in Amelanchier laevis, Shadbush (Rosaceae, Maloideae). Amer. J. Bot. 72: 1397-1403.

GoRCHOV, D. L. 1985. Fruit ripening asynchrony is related to variable seed number in Amelanchier and Vaccinium. Amer. J. Bot. 72: 1939-1943.

ㄴ. 1987. Proximate and ultimate causes of fruit ripening asynchrony in vertebrate-dispersed woody plants in southeastern Michigan. Ph.D. dissertation, University of Michigan, Ann Arbor.

Harper, J. L. 1977. Population biology of plants. Academic Press, London.

Herrera, C. M. 1981. Fruit variation and competition for dispersers in natural populations of Smilax aspera. Oikos 36: 51-58.

LeE, T. D., AND F. A. BazzAz. 1982. Regulation of fruit maturation pattern in an annual legume, Cassia fasciculata. Ecology 63: 1374-1388.

Olson, A. R., AND T. A. Steeves. 1982. Structural changes in the developing fruit wall of Amelanchier alnifolia. Canad. J. Bot. 60: 1880-1887.

RATHCKE, B. 1983. Competition and facilitation for pollination. In L. Real [ed.], Pollination biology, 305329. Academic Press, New York.

Robinson, W. A. 1982. Experimental taxonomy in the genus Amelanchier II: Do the taxa in the genus $A m e-$ lanchier form an agamic complex? Rhodora 84: 85100.

- 190. 1986. Effect of fruit ingestion on Amelanchier seed germination. Bull. Torrey Bot. Club 113: 131134.

SNOw, A. A. 1986. Pollination dynamics in Epilobium canum (Onagraceae): consequences for gametophytic selection. Amer. J. Bot. 73: 139-151.

Stephenson, A. G. 1981. Flower and fruit abortion: proximate causes and ultimate functions. Annu. Rev. Ecol. Syst. 12: 253-279.

Thompson, J. N., AND M. F. WILlson. 1979. Evolution of temperate fruit/bird interactions: phenological strategies. Evolution 33: 973-982.

Wareing, P. F., AND I. D. J. Phillips. 1978. Control of growth and differentiatin in plants. $2 \mathrm{~d}$ ed. Pergamon Press, Oxford and New York. 